\title{
Summary of SC TCM Agricultural Soil Ecological Restoration Major Scientific and Technological Innovation Engineering Technology
}

\section{Hongfeng Sun* Hongwei Luan}

Beijing World Science and Technology Research and Development Center for Productivity, Beijing, 102600, China

\begin{tabular}{l}
\hline ARTICLE INFO \\
\hline Article history \\
Received: 19 March 2020 \\
Revised: 26 March 2020 \\
Accepted: 24 April 2020 \\
Published Online: 30 April 2020 \\
\hline
\end{tabular}

Keywords:

SC TCM

Agricultural soil ecological restoration

Technological innovation engineering

\section{Introduction}

0 C TCM agricultural soil ecological restoration major scientific and technological innovation project is the chairman of the executive committee of the international academician consortium, commended by the United Nations WCPS organization, and won the "100 outstanding inventors of the 60th anniversary of the founding of the People's Republic of China" and "Productivity Science of the 70th anniversary of the founding of the People's Republic of China" "Field Meritorious Scientist" honorary title, founder of "Plant Nutritional Growth and Reproductive Growth Balance Theory", world-famous scientist known as "Father of Fertilizers in China", Academician Sun Cheng of World Academy of Productivity Science (WAPS) and Chen Yanxi of China Agricultural University, Mei Ruhong, Wang Qi and other academicians and experts and their teams developed the latest major scientific and tech-

\section{ABSTRACT}

The paper focuses on the major scientific and technological innovation engineering technology of SC TCM agricultural land ecological restoration. The core technology system of SC TCM agricultural soil ecological restoration major scientific and technological innovation project consists of a number of cutting-edge advanced technologies from four aspects: "Century Tianwang" high-concentration nano-bio-organic fertilizer, high-concentration nano-bio-organic-inorganic slow-release compound fertilizer, "Century Tianwang" nano-soil special repair material; "Century Tianwang" botanical pesticide residue degradation agent; SOD rumex planting and breeding cycle technology.

nological achievements in soil ecological restoration. Among them, "saline-alkali soil fertilizer and salt balance bio-nano improvement technology" has been listed as a key research and development project of Shandong Province in 2019.

TCM agriculture received the focus of the United Nations Food and Agriculture Organization (FAO) at the 15th World Congress of Traditional Chinese Medicine. The SC TCM agricultural soil ecological restoration project is a major scientific and technological innovation project that adopts the principles and methods of TCM and cross-border integration of agriculture. Academician Sun Cheng's major invention patent technology achievement, listed as the national key new product plan project and the national high-tech industry key demonstration project, the upgrade of the national key new product plan project, and the innovation; it is the professional invention patent technology of academician Sun Cheng The brand "Century Tianwang" high-concentration na-

*Corresponding Author:

Hongfeng Sun,

Beijing World Science and Technology Research and Development Center for Productivity, 102600, China;

E-mail: wcpscc@vip.sina.com 
no-bio-organic fertilizer, nano-biological soil special repair agent, nano-plant-derived pesticide residue degradation agent, basic particle microecological preparation, SOD sorrel planting and breeding cycle science is deeply integrated, producing a huge comprehensive effect in the world The latest high-end technological achievements. It is the resource utilization of organic solid wastes such as livestock manure, straw, sludge, domestic garbage, and agricultural and sideline products that have undergone harmless treatment. It is the comprehensive practical application of the technical achievements of a number of invention patents such as "Century Tianwang" bio-organic fertilizer by Academician Sun Cheng, "a new technology for production of eco-friendly and environmentally friendly compound fertilizer controlled and slow-released", "a contaminated soil remediation composition", etc., has formed industrialization. More than 30 enterprises across the country have introduced the technological achievements and established the "Century Tianwang" production line, which is now called "Hubei Fengyi Fertilizer Co., Ltd.", "Hubei Ruidi Biological Technology Co., Ltd., Companies such as "Beijing Gutian Fertilizer Co., Ltd." and "Jiangxi Qijia Fertilizer Co., Ltd." have formed the largest organic-inorganic slow-release compound fertilizer enterprise alliance in China, the "Century Tianwang Enterprise Alliance".

The core technology system of SC TCM agricultural soil ecological restoration major scientific and technological innovation project consists of a number of cutting-edge advanced technologies, mainly including.

\section{2 "Century Tianwang" High-Concentration Nano-Bio-Organic Fertilizer, High-Con- centration Nano-Bio-Organic-Inorganic Slow-Release Compound Fertilizer}

\subsection{Main Features}

"Three Nos", "Three Highs", "Two Completes" and "One Degradation".

(1) "Three Noes": non-toxic, harmless, and odorless;

(2) "Three Highs": The product contains high nutrients, high organic matter, and high humic acid;

(3) "Comprehensiveness": fertilizer has all nutrients and fertilizer functions;

(4) "One degradation": It can degrade chemical pesticide residues.

\subsection{Main Technical Routes and Innovation Points}

(1) Nano slow-release technology is combined with agricultural microbial technology. Fertilizer products contain a multi-functional nano-organic compound integrating urease inhibition, nitrification inhibition, ammonia stabilization and plant growth regulation, and a special effect "ST highly concentrated compound microbial bacteria", which can greatly improve fertilizer nutrient utilization, Reduce the salt index in fertilizers, adjust $\mathrm{PH}$ value, stimulate crop growth and enhance crop resistance.

(2) Combination of organic and inorganic. Improve soil organic matter, improve soil aggregate structure, increase crop organic matter, and reduce agricultural environmental pollution.

(3) Integration of medicine and fertilizer. Add "pesticide degradation antidote" and Yiwei SOD microecological preparation extracted from pure natural plant source Chinese herbal medicine to fertilizer to kill insects and bacteria, and degrade pesticide residues.

(4) Advanced, scientific and reasonable formula. According to the theory of "yin and yang balance" and "nutrition balance" in traditional Chinese medicine, following the balanced proportional relationship between plant nutrition growth and reproductive growth, design advanced, scientific and reasonable fertilizer formulas for various crops.

\subsection{Application Effect}

(1) The fertilizer utilization rate is increased by more than $30 \%$;

(2) The amount of chemical fertilizer applied is reduced by $30 \%-40 \%$;

(3) The utilization rate of organic resources is increased by $30 \%-40 \%$;

(4) Improve soil organic matter by $5 \%-10 \%$;

(5) Increase the yield of grain crops by $10 \%-20 \%$; increase the yield of vegetables, fruits and cash crops by 20\%-30\%;

(6) $90 \%-100 \%$ degradation of pesticide residues; reduction of pesticide application by $80 \%-90 \%$;

(7) The land output rate is increased by more than $30 \%$;

(8) Reduce agricultural fertilizer costs by $30 \%-50 \%$;

(9) The fertilizer salt index is low, and the application is safe and reliable;

(10) Strengthen crop resistance, drought resistance, cold resistance, water saving, lodging resistance, prevention and control of various diseases and insect pests.

\section{3 "Century Tianwang" Nano-Soil Special Re- pair Material}

"Century Tianwang" nano-soil special remediation materials, including "saline-alkali land special remediation materials", "heavy metal contaminated farmland 
special remediation materials", "petroleum contaminated farmland special remediation materials", "desertified land special remediation materials", "secondary contaminated land" "Special repair materials" series of special soil repair materials.

"Century Tianwang" special nano-remediation material for soil is the latest major issue that has been successfully developed for a series of major issues such as the improvement of saline-alkali land, the treatment of heavy metal contaminated farmland, the treatment of oil-contaminated farmland, the restoration of secondary polluted farmland, desertified land, and the restoration of compacted soil with reduced organic matter Technological Achievements.

"Century Tianwang" special nano-remediation material for soil is a contaminated soil remediation composition. It is composed of a natural nanometer that integrates nitrification inhibition, urease inhibition, ammonia stabilization, pest control, disease elimination, pesticide residue reduction, and plant growth regulation. Organic compounds, "Century Tianwang" fertilizer nano slow-release agent, "ST high-efficiency concentrated compound microbial special fungus", "Yiwei SOD microecological preparation" "Native natural humic acid", Chinese herbal medicine plant source extracts and nano-functional nutrient materials It is the basic raw material, processed by a specific process.

"Century Tianwang" special nano-remediation material for soil is a special high-tech material for soil remediation, chemical pesticides, fertilizer reduction and efficiency enhancement. It is a major invention patent technical achievement of Academician Sun Cheng and a national key new product plan project "Century Tian The core technology material of "Wang Bio-Organic Fertilizer". The restoration material is not only pollution-free and residue-free, but also effectively degrades the original pesticide residues in the soil, removes harmful metals, and improves soil organic matter.

"Century Tianwang" soil-specific nano-remediation materials have special effects on the transformation of saline-alkali soils. It can improve the soil's ability to adsorb molecular and ionic substances, promote the combined force of fertilizer and salt balance to adsorb and transform salt, effectively reduce the salt index, reduce salt content, adjust acid-base balance, improve soil salt resistance, and improve the soil structure of saline-alkali soils. Improve soil organic matter.

"Century Tianwang" soil-specific nano-remediation materials play an important role in the restoration of heavy metal-contaminated farmland and the treatment of desertified land. Mainly manifested in that, on the one hand, it can directly interact with the heavy metal ions in the soil physically and chemically, affecting their form, migration, transformation and biological effectiveness in the environment, thereby fixing heavy metals and reducing their activity; on the other hand, applying to the soil After that, it can effectively improve soil structure and properties, such as soil organic matter content and $\mathrm{PH}$ value, and improve the soil's own buffer and fixation capacity for heavy metals. In addition, due to the increase of organic matter content, it promotes the formation of soil aggregates and water storage, water retention, and fertilizer retention, which have special effects on desertified land management.

Among the soil remediation materials, the natural plant-derived extracts of Chinese herbal medicines have special effects on soil deworming and detoxification, effectively degrading residual pesticides such as organic phosphorus, carbamates, and sulfonylureas in the soil, with a degradation rate of over $90 \%$.

"Century Tianwang" special nano-remediation material for soil, matched with agricultural fertilizers, and applied at the same time, has a huge effect on fertilizer reduction and efficiency. Compared with the traditional application of chemical fertilizers and compound fertilizers, the application amount of chemical fertilizers and compound fertilizers can be reduced by more than $50 \%$. Most types of crops can be exempted from pesticides, and some special fruit and vegetable crops can also be reduced by more than $80 \%$. Food crops can increase production by more than $10 \%$, and vegetables and fruits can increase production by $20 \%$ to $30 \%$. The reason why it can achieve the effect of reducing fertilization and increasing efficiency is mainly because the "Century Tianwang" soil special nano-remediation material, when used in conjunction with fertilizers, can enable plants to perform photosynthesis, chlorophyll synthesis, protein synthesis, and enzyme activities during the day. In good condition, it promotes the metabolism and growth of plants; the nano-compounds in the soil-specific nano-release agent have strong ammonia-fixing ability, which can effectively inhibit the formation of nitrate nitrogen in the soil, inhibit the activity of nitrifying bacteria, and increase soil colloids The adsorption strength of the clay particles to ammonia ions and sodium ions can not only reduce the salt-alkali concentration, but also enable the slow release of nutrient elements in the soil, reduce nutrient element loss, improve fertilizer utilization, and meet plant nutrient elements. Soil-specific nano-remediation materials are used in conjunction with fertilizers. Due to the action of microorganisms and humic acid, they not only effectively increase soil organic matter, 
improve soil structure, and promote plant growth, but also increase the activity of crop catalase and reduce cell membrane permeability. And abscisic acid content, thereby enhancing crop resistance, flood resistance, drought resistance, cold resistance, lodging resistance, resistance to diseases and insect pests.

\section{4 "Century Tianwang" Botanical Pesticide Residue Degradation Agent}

As an important means of agricultural production, chemical pesticides play an important role in curbing plant diseases, insect pests and weeds and reducing agricultural production losses. However, long-term excessive and unreasonable application of chemical pesticides has also caused serious impacts on human production, life and the ecological environment. The resulting quality and safety of agricultural products, decline in the quality of cultivated land, agricultural non-point source pollution, sudden increase in malignant diseases and environmental hormone problems have caused highly anticipated.

Organophosphorus pesticides are widely used, especially in the field of pesticides, accounting for more than $70 \%$ of the total pesticides. The utilization rate of pesticides applied in farmland is $38.8 \%$. Most of the pesticides diffuse into the environment, enter the human body through the water vapor cycle and the food chain, and endanger health. Moreover, because a large amount of pesticides remain in the soil, they have become an important factor in agricultural non-point source pollution. One.

Pesticide degradation can be divided into physical methods, chemical methods, and biological methods. However, due to factors such as application conditions, use costs, and technological maturity, it has not been promoted and applied on a large scale, especially in agricultural production. There is no precedent. At present, biological methods are considered to be an ideal method to reduce pesticide residues due to their significant effect of degrading pesticide residues and low environmental impact. In particular, the screening of pesticide degrading bacteria and the research and development of application technologies have achieved significant research results, but these technologies still in the laboratory research and development stage.

"Century Tianwang" botanical pesticide residue degradation agent, is a team member of the famous scientist Academician Sun Cheng, researcher Sun Jun, and Dr. Zhang Tongxin. It took 12 years to develop a major invention patent technology achievement.

"Century Tianwang" botanical pesticide residue degradation agent, based on the principle of enzymatic deg- radation of pesticides, pioneered a unique way, based on the theory of traditional Chinese medicine, prescriptions and years of application practice, to screen and develop plant sources with rapid and efficient degradation effects on organophosphorus pesticides The active ingredients, through indoor testing and field verification, have significant effects, realize the cross-border integration of modern agriculture and traditional Chinese medicine, and provide a good technical path and basic data support for exploring and applying the concept of Chinese medicine to promote the harmonious development of "production, life and ecology". It has important scientific research and application value.

"Century Tianwang" botanical pesticide residue degradation agent, through the mass production and field practice application cases of Shandong Rongjian Biotechnology Company, has conducted field tests on a large area of various crops for many years, and it has shown that it is effective against organophosphorus and carbamates. And sulfonylurea (herbicide) pesticides have a significant degradation effect, and the degradation rate is extremely fast. The degradation rates of chlorpyrifos and parathion organophosphorus pesticides reached $93.2 \%$ and $92.9 \%$ within 2 minutes; the degradation rates of aldicarb and carbofuran amino acid lipid pesticides reached $87.5 \%$ and $85.1 \%$; passed the pesticide the residue rapid test method showed that the degradation rate of dichlorvos within $17 \mathrm{~h}$ was $66.67 \%$, and the degradation rate of chlorpyrifos was $48.69 \%$ within $11 \mathrm{~h}$.

The application of "Century Tianwang" botanical pesticide residue degradation agent has great application value in the fields of soil remediation, agricultural product safety, water body and environmental biological clinics, and it also provides a new technological path for the rapid degradation of chemical pesticides. . The use of plant enzyme clusters to rapidly degrade chemical pesticides completely solves the problems of soil pesticide residue pollution and pesticide residues in ground products, and creates a new era of green ecological agriculture.

Detoxification of contaminated farmland soil is the most critical first step in soil remediation. The remaining chemical pesticides are not eliminated, which will seriously affect the survival rate and biological activity of the microbial flora, resulting in a significant decrease in the repair effect of subsequent use of biological bacterial fertilizer and organic fertilizer. And "Century Tianwang" botanical pesticide residue degradation agent and "Century Tianwang" high-concentration nano-bio-organic fertilizer, high-concentration bio-organic-inorganic slow-release compound fertilizer, soil bio-nano-special repair agent and other products are used in a complementary manner. 
Formed the "SC TCM agricultural soil ecological restoration new technology" system to achieve the best effect of soil restoration. It can not only degrade pesticide residues, but also improve soil organic matter, increase crop yields, improve crop quality, and realize modern ecological agriculture Green, environmentally friendly, economical and efficient.

\section{SOD Rumex Planting and Breeding Cycle Technology}

The SOD sorrel planting and breeding cycle technology is a huge achievement made by academicians and experts of China Agricultural University such as Chen Yanxi, Mei Ruhong, Wang Qi and their teams. The cumulative application area in China has reached 1.5 billion mu. Team members Wang Qi, Chen Bi, Mei the three professors of Ruhong were awarded the commemorative medals for "Celebrating the 70th Anniversary of the Founding of the People's Republic of China".

The implementation of the SC TCM agricultural soil ecological restoration major scientific and technological innovation project is aimed at the practical problems of large investment, slow response, and low efficiency in the restoration of contaminated land such as saline-alkali and desertified land. The SOD sorrel planting and breeding cycle technology is used to plant high-SOD-tolerant plants. The sorrel has a return that year and produces higher economic benefits.

There are wild sorrel in all parts of northern China. Its scientific name is sorrel, nicknamed sorrel leaves. It can be used as Chinese herbal medicine. The sorrel in this project is a hybrid variety introduced by China from Ukraine. It is cultivated and selected by the international academician consortium and the team of academicians of the Institute of Plant Ecological Engineering of China Agricultural University. It has a high content of superoxide dismutase SOD and has high added value. The latest plant varieties.

The effects of sorrel against saline and alkali and improving soil are mainly manifested in:

One is to adjust the $\mathrm{pH}$ value of the soil, the huge root metabolism of Rumex sorrel, a large number of stems and leaves fall into the soil, after decomposition, under the action of the rhizosphere microorganisms and rhizosphere microorganisms of sorrel, various organic acids are produced, which play a role in soil alkalinity Neutralize and reduce $\mathrm{PH}$ value;

The second is to change the water and salt conditions and accelerate soil desalination. The soil salt balance depends on the growth and decline of evaporation and leaching capacity. When the increased amount is greater than the leaching amount, salt accumulates, otherwise desalination occurs. The luxuriant clusters of sorrel leaves basically cover all the ground, which can change the field microclimate and reduce surface water evaporation and salt accumulation.

The third is to increase the soil aggregate structure and the number of microorganisms. The beneficial microorganisms in the root system of sorrel, such as nitrifying bacteria and aerobic fiber decomposing bacteria, have increased significantly;

The fourth is to increase soil nutrient content. Due to the well-developed root system of sorrel, the biological yield of the above-ground part of sorrel is high, the number of sorrel residues remaining in the soil is also large, and the content of soil organic matter has increased significantly. The soil organic matter of sorrel planted for 4 years can increase by about $0.5 \%$. In addition to high protein, lysine, superoxide dismutase, flavonoids and other nutrients, sorrel is also rich in other nutrients;

The fourth is to increase soil nutrient content. Due to the well-developed root system of sorrel, the biological yield of the above-ground part of sorrel is high, the number of sorrel residues remaining in the soil is also large, and the content of soil organic matter has increased significantly. The soil organic matter of sorrel planted for 4 years can increase by about $0.5 \%$. In addition to high protein, lysine, superoxide dismutase, flavonoids and other nutrients, sorrel is also rich in other nutrients;

Fifth, inoculation of Rumex with high SOD-containing endosymbiont bacillus has a unique effect. SOD can effectively remove free radicals in organisms and can disproportionate toxic oxygen free radicals into non-toxic water and oxygen;

Sixth, reduce the salt index, adjust the osmotic pressure of the soil solution, and enhance crop resistance.

Rumex itself was introduced into China from Ukraine as feed. It has been approved by the Global Forage Variety Approval Committee and registered as a nationally introduced species in China with registration number 183.

Sorrel is the most outstanding forage species and the latest forage species with high yield, new type and high protein. The crude fat content of sorrel is significantly higher than that of general pasture, leafy vegetables and stem vegetables. The crude fat content of dry matter is $6.21 \%$, while that of alfalfa is only $3 \%$, spinach is $3 \%$, and cowpea is $2 \%$.

The content of crude fiber, vitamins, and minerals in sorrel are also higher than other pasture and vegetable plants.

It can be seen that sorrel is a high-quality protein 
source feed. When changing the saline-alkali land and desertified land, a large number of sorrel is planted and processed into green silo feed and pelleted protein feed to supply the livestock and animal breeding industry, which is conducive to promoting the economic development of recycling agriculture. Rumex can not only be deep-processed into SOD-rich high-protein feed, allowing people to eat healthy meat, eggs, milk and other animal functional foods, but also, because SOD is currently recognized as the only beneficial and harmless antioxidant in the world, it is Harmful free radical killer. Therefore, the SC TCM agricultural soil ecological restoration academician expert team has the conditions for soil ecological restoration and planting sorrel. According to needs, sorrel SOD extraction technology and sorrel inoculation SOD inoculant technology can be used to produce SOD-rich apples and pears. Various SOD fruits such as, peach, strawberry, corn, rice, wheat and other SOD-rich food crops and various SOD beverages, enhance human immunity, improve human health, and contribute to the construction of a human public health and health community.

\section{References}

[1] Xiao Fan. "Traditional Chinese Medicine Agriculture" unlocks new agricultural science and technology skills [J]. China Rural Science and Technology, 2018:.36-37.

[2] Meng Xia. "Traditional Chinese Medicine Agriculture" opens up a new path of characteristic ecological agriculture [J]. Farmer Science and Technology Training, 2017(02): 38-39.

[3] Wang Yanqun. Research on the technology and effect of soil ecological environment restoration in facility farmland [D]. Baoding: Hebei Agricultural University, 2005. 